\title{
A whole-body physiologically based pharmacokinetic (WB-PBPK) model of ciprofloxacin: a step towards predicting bacterial killing at sites of infection
}

\author{
Muhammad W. Sadiq ${ }^{1,2}$ - Elisabet I. Nielsen ${ }^{1}$ - Dalia Khachman ${ }^{3,4}$. \\ Jean-Marie Conil ${ }^{5,6} \cdot$ Bernard Georges $^{5,6}$ - Georges Houin ${ }^{5}$ Celine M. Laffont ${ }^{3,4}$. \\ Mats O. Karlsson ${ }^{1} \cdot$ Lena E. Friberg ${ }^{1}$ (D)
}

Received: 1 June 2016/Accepted: 18 August 2016/Published online: 30 August 2016

(C) The Author(s) 2016. This article is published with open access at Springerlink.com

\begin{abstract}
The purpose of this study was to develop a whole-body physiologically based pharmacokinetic (WBPBPK) model for ciprofloxacin for ICU patients, based on only plasma concentration data. In a next step, tissue and organ concentration time profiles in patients were predicted using the developed model. The WB-PBPK model was built using a non-linear mixed effects approach based on data from 102 adult intensive care unit patients. Tissue to plasma distribution coefficients $(\mathrm{Kp})$ were available from the literature and used as informative priors. The developed WB-PBPK model successfully characterized both the typical trends and variability of the available ciprofloxacin plasma concentration data. The WB-PBPK model was thereafter combined with a pharmacokinetic-pharmacodynamic (PKPD) model, developed based on in vitro timekill data of ciprofloxacin and Escherichia coli to illustrate the potential of this type of approach to predict the timecourse of bacterial killing at different sites of infection. The
\end{abstract}

Electronic supplementary material The online version of this article (doi:10.1007/s10928-016-9486-9) contains supplementary material, which is available to authorized users.

Lena E. Friberg

lena.friberg@farmbio.uu.se

1 Department of Pharmaceutical Biosciences, Uppsala University, Box 591, 75124 Uppsala, Sweden

2 CVMD iMED, DMPK, Astrazeneca, Mölndal, Sweden

3 INRA, Toxalim, Toulouse, France

4 Universite de Toulouse, Toulouse, France

5 Laboratoire de Pharmacocinetique et Toxicologie Clinique, Hospital Purpan, Institut Federatif de Biologie, Toulouse, France

6 Pole d'Anesthesie-Reanimation, Hopital Rangueil, Toulouse, France predicted unbound concentration-time profile in extracellular tissue was driving the bacterial killing in the PKPD model and the rate and extent of take-over of mutant bacteria in different tissues were explored. The bacterial killing was predicted to be most efficient in lung and kidney, which correspond well to ciprofloxacin's indications pneumonia and urinary tract infections. Furthermore, a function based on available information on bacterial killing by the immune system in vivo was incorporated. This work demonstrates the development and application of a WBPBPK-PD model to compare killing of bacteria with different antibiotic susceptibility, of value for drug development and the optimal use of antibiotics .

Keywords Physiologically-based pharmacokinetic . Antibiotic · Modeling - Bacterial infection . Pharmacokinetic-pharmacodynamic · Fluoroquinolone . Informative priors · NONMEM

\section{Introduction}

The time-course of the distribution of an antibiotic into an infected tissue can be of critical importance for successful therapy of the infection. Based on the physicochemical properties of a drug and its interaction with different transporters, the rate and extent of distribution differs between tissues $[1,2]$. Sub-therapeutic exposure of an antibiotic at the site of infection may not only result in treatment failure but also emergence of resistance, while higher than therapeutic levels may result in toxicity. Typically, when pharmacokinetic-pharmacodynamic (PKPD) relationships of antibiotics are evaluated, the bacterial killing is assumed to be dependent on a summary variable of the pharmacokinetic (PK) profile in plasma (e.g. peak 
plasma concentration, area under the plasma concentration-time curve or time period that the plasma concentration exceeds the MIC) [3]. Using the plasma concentration to monitor and drive the antimicrobial effect is practical, but the plasma concentration is not always reflecting the biophase antimicrobial concentration. In addition, the dynamics of the concentrations during the initial treatment period may be critical for a successful outcome. Overlooking that the time-course of concentration in the infected tissue is what is driving the bacterial killing could lead to suboptimal dosing in patients. In the era of increasing antibiotic resistance, new therapies need to be explored for previously untested types of infections. To efficiently get an idea of the potential of these therapies it is important to understand the distribution and exposure time profile of antimicrobials in the target tissues.

Whole body physiologically based pharmacokinetic (WB-PBPK) models encompass the distinct feature of describing the distribution of a drug in different tissues and blood in a mechanistic way [4-6]. Such models have long been used in toxicological risk assessment for different environmental contaminants [7]. In recent years they have been increasingly applied in different stages of drug discovery and development, and also recognized by regulatory authorities as a valuable tool [1, 7-12]. WB-PBPK models require two types of input data; physiological parameters such as blood flows, organ volumes, tissue compositions and drug related parameters like plasma protein binding, clearance and tissue to plasma partition coefficients (Kp). The $\mathrm{Kp}$ values reflect the distribution in the tissue in comparison to plasma at steady state. It is tedious, time and resource extensive to measure these values for each tissue experimentally, and based on the physicochemical properties of a compound there are different methods proposed to predict Kp values [10, 13-15]. Valuable information on Kp values may also be collected from in vivo experiments reported in the literature especially for antibiotics which are used prophylactically before soft tissue surgery due to different pathological indications.

During recent years, there have been different PKPDmodels proposed to understand and quantify the timecourse of bacterial killing $[3,16]$. Such models may subsequently be linked to a PK model to predict the bacterial killing following different antibiotic dosing regimens $[17,18]$. In this way, complex kinetic profiles, such as multiple compartment kinetics (e.g. gentamicin) and formation from prodrugs (e.g. colistin) can be mimicked without a range of extensive new time-kill experiments. Typically, the unbound concentration in the central compartment (e.g. plasma) has been used to drive the killing of the bacteria in the PKPD-models, i.e. a sepsis situation has been simulated.
In a patient the clinical outcome of the therapy will also be influenced by the presence of the immune system. Guo et al. has proposed a model for the quantitative impact of neutrophils on bacterial clearance in mice where the bacterial killing was dependent on the grade of immunosuppression, i.e. the absolute neutrophil count [19]. Further, Drusano et al. have shown in mice that the bacterial clearance by the immune cells is saturable and a model was proposed where the bacterial killing was dependent on the bacterial burden [20]. The time-course of the efficiency of the immune system to clear the infection is however most likely dependent on both the number of bacteria at the site of infection and the competence of the immune system.

Ciprofloxacin belongs to the fluoroquinolone class of antimicrobials and has been extensively used the last two decades in the clinic because of its broad spectrum against bacteria [21]. Fluoroquinolones are associated with rapid emergence of resistance and hence appropriate therapeutic use of this class is prime to keep their effectiveness. As ciprofloxacin has been used for many years it is also possible to collect information on $\mathrm{Kp}$ values based on clinical samples. Therefore, ciprofloxacin can serve as an example on how in vitro, in vivo and literature information can be integrated into a whole-body PBPK model to predict unbound concentrations in different tissues and organs. Recently a PKPD model was developed by Khan et al. based on data from time-kill experiments with constant ciprofloxacin experiments [22]. The model describes the growth and killing kinetics of a wild-type Escherichia coli strain, and six well-characterized mutants thereof, with different degree of resistance, during exposure to various ciprofloxacin concentrations.

In this study we aimed to develop a WB-PBPK model for predictions of the tissue/organ concentration-time profiles of ciprofloxacin in patients. In a next step the model was combined with a PKPD model, describing bacterial growth and ciprofloxacin killing kinetics, as well as a model characterizing the effect of the immune system. The combined model was used to illustrate the potential value of this approach to predict the time-course of bacterial killing for infections with E. coli strains with different levels of resistance.

\section{Methods}

\section{WB-PBPK model building}

A WB-PBPK model for ciprofloxacin was developed based on plasma concentration data from 102 adult patients admitted to the Intensive Care Unit (ICU) for different indications. The data has earlier been used to develop a 2-compartment population PK model [23]. There were 27 
Fig. 1 Structure of the WBPBPK model developed for ciprofloxacin. Volumes of different tissue compartments $(V)$ and blood flows to tissues $(Q)$ were described on the basis of individual weight and gender

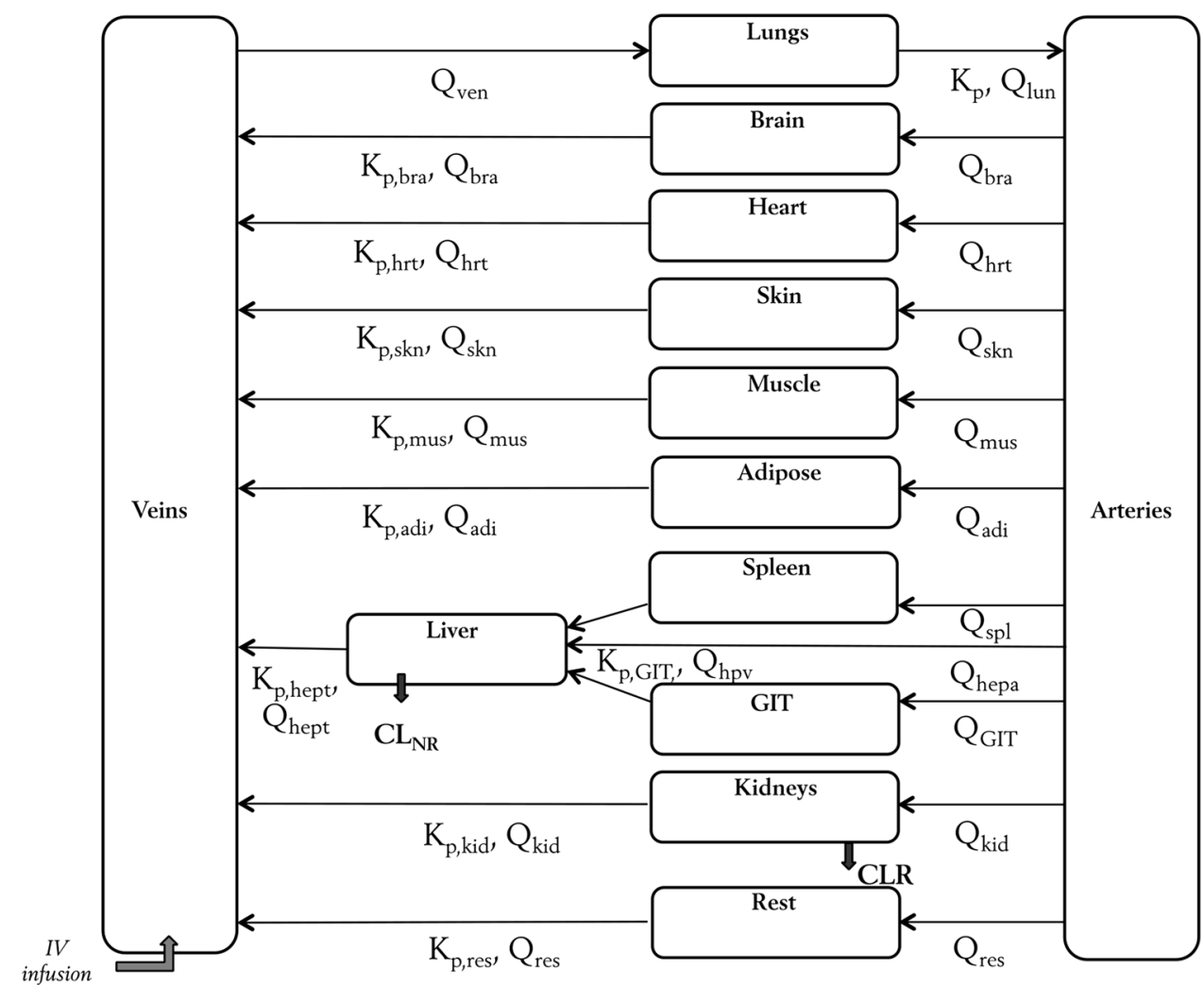

women and 75 males in the patient population with an average \pm SD total body weight of $77 \pm 16 \mathrm{~kg}$ and an average age of $60 \pm 17$ years. Measured creatinine clearance (CRCL) was $82 \pm 51 \mathrm{ml} \mathrm{min}^{-1}$. All patients were mechanically ventilated and were on ciprofloxacin infusion therapy during their stay in the ICU. Among the 102 patients included in the study 86 received $400 \mathrm{mg}$ ciprofloxacin as a $1 \mathrm{~h}$ infusion twice a day, 9 patients received $400 \mathrm{mg}$ three times a day, 6 patients received $200 \mathrm{mg}$ twice a day (30 min infusion), and 1 patient received $600 \mathrm{mg}$ twice a day [23]. Duration of treatment ranged from 3 to 21 days (average $=12$ days). In total 588 plasma concentrations were available, with on average 5.8 samples from each patient. Samples were taken on different days where one occasion was defined to be one dose administration interval (average $=3.1$ occasions/patients).

A population modeling approach was applied to fit WBPBPK models to the plasma PK data using NONMEM version 7.3 with first order conditional estimation [24]. All plasma concentrations from patients were transformed into natural logarithms before the data analysis. An additive error model was used on log-transformed data. During the estimation process the only dependent variable was the plasma concentrations.

Typical population ciprofloxacin tissue to plasma distribution coefficients $(\mathrm{Kp})$ for 10 different tissues including lung, muscle, kidney and adipose were taken from clinical studies available in the literature and used as informative priors while estimating the tissue $\mathrm{Kp}$ values [25-32]. Kp values are determined from the ratio of total tissue and plasma concentrations and described according to Eq. (1).

$\mathrm{K}_{\mathrm{p}}=\mathrm{C}_{\text {Tissue }} / \mathrm{C}_{\text {Plasma }}$

when a $\mathrm{Kp}$ value was not directly reported from a study (spleen, heart and brain) the value was calculated by using the ratio of the reported tissue and plasma concentrations. A "Rest" compartment represented other parts of the body for which no Kp is defined, and for this compartment the typical $\mathrm{Kp}$ was set to the average of all $\mathrm{Kp}$ values for other tissues.

A frequentist prior approach, using the normal-Wishart prior subroutine (NWPRI) in NONMEM, was applied. With this approach, uncertainty in the prior Kp values can be considered in the estimation. An uncertainty of $25 \%$ around the typical prior values was applied for each tissue and implemented as a normal distribution on log-scale [33]. A generic structure of a WB-PBPK model was adopted assuming perfusion limited drug distribution in all tissues (Fig. 1) [34]. Different physiological parameters including blood flow to different organs (Q) as well as organ volumes (V) were taken from the literature [35, 36], and incorporated in the model as a function of weight and gender of the individual patient. Instead of using the typical weight of $70 \mathrm{~kg}$ individual patient weights were used to calculate the 
volume of different tissues as fraction of total body weight. Gender based differences in the body composition were also accounted for when defining the tissue volumes and blood flows (see NONMEM code in online appendix) $[35,36]$.

Clearance (CL) of ciprofloxacin was divided into renal and non-renal clearance in the WBPBPK model (Eq. 2). For a typical patient with a CRCL of $120 \mathrm{ml} \mathrm{min}^{-1}$ approximately $45 \%$ of ciprofloxacin is excreted by the kidneys [37], resulting in a prior mode for non-renal clearance $\left(\mathrm{CL}_{\mathrm{NR}}\right)$ of $7.17 \mathrm{~h} \mathrm{~h}^{-1}$ and for fraction secretion $\left(\mathrm{f}_{\text {secretion }}\right)$ of 0.57 . Individual CRCL values were used to calculate the renal clearance $\left(\mathrm{CL}_{\mathrm{R}}\right)$ according to Eq. (3)

$\mathrm{CL}=\mathrm{CL}_{\mathrm{R}}+\mathrm{CL}_{\mathrm{NR}}$

$\mathrm{CL}_{\mathrm{R}}=\mathrm{CRCL} \times \mathrm{f}_{\mathrm{u} \text {,plasma }} \times\left(1+\mathrm{f}_{\text {Secretion }}\right)$

where $f_{u, p l a s m a}$ is the fraction unbound of ciprofloxacin in plasma ( $f u=0.65)$ [37] and $f_{\text {Secretion }}$ is the here estimated fraction of the ciprofloxacin renal clearance that is dependent on secretion from blood to renal tubules. Unexplained inter-individual variability (IIV) was explored without applying a prior.

The differential equations describing the mass transfer in the WBPBPK model are illustrated in the NONMEM code (see online appendix). CL and Kp values were estimated on the log-scale.

\section{Model predictions}

During all predictions the doses of ciprofloxacin was kept at $400 \mathrm{mg}$ b.i.d. and administered intravenously as a $1 \mathrm{~h}$ infusion. To predict unbound ciprofloxacin concentrationtime profiles in different tissues, the total concentrations of ciprofloxacin predicted by the WB-PBPK model were converted to unbound concentrations by using Eq. (4) where the unbound fraction in extracellular fluid (fue) is defined [38].

fue $=1 /(1+\mathrm{E} / \mathrm{P} \times(1-\mathrm{fu}$, plasma $) /$ fu, plasma $)$

$\mathrm{E} / \mathrm{P}$ is the ratio of albumin in extracellular fluid to plasma. $\mathrm{E} / \mathrm{P}$ values for each organ or tissue was taken from literature values reported for rats [14].

The final WB-PBPK model was coupled to a PKPD model of ciprofloxacin for E. coli [22] to quantitatively predict the time-course of the bacterial killing in the different tissues. The details of the PKPD model have earlier been described and the differential equations are included in the NONMEM code (online appendix). In brief, the model includes parameters for natural growth $\left(1.70 \mathrm{~h}^{-1}\right)$ and death $\left(0.179 \mathrm{~h}^{-1}\right)$, a structure that allows for characterization of a maximum bacterial concentration, an $\mathrm{E}_{\max }{ }^{-}$ model for the ciprofloxacin-induced rate of killing, the presence of a less susceptible subpopulation ( 0.819 bacteria per $10^{6} \mathrm{CFU} \mathrm{ml}{ }^{-1}$ start inocula), and a $5.34 \mathrm{~h}$ time period where the bacteria were susceptible to ciprofloxacin-induced filament formation and hence not countable when plated. The predicted unbound ciprofloxacin concentrations in the extracellular space of the various tissues and organs were in this step used to drive the concentration-dependent rate constant of bacterial killing. The extracellular concentrations were used to drive the effect due to the fact that $E$. coli are assumed to be in the extracellular space. Predictions were made for different $E$. coli strains (LM347, LM202, LM378, LM625, LM693 and LM707) of variable resistance levels (MIC ranging from 0.023 to $48 \mathrm{mg} \mathrm{l}$ ). When two strains were combined to explore the rate and extent of mutant take-over, each strain had a starting bacteria concentration of $5 \cdot 10^{6} \mathrm{CFU} \mathrm{ml}{ }^{-1}$.

In a next step, the effect of the immune response on the bacterial clearance was added. In accordance to previous publications $[19,20]$, the killing by neutrophils was here assumed to be dependent on both the neutrophil count and the number of bacteria in the tissue and a term (Eq. 5) for the immune response was added to all bacterial compartments (see online appendix)

$$
\begin{aligned}
\mathrm{dB} / \mathrm{dt}= & \ldots-\text { Kkill_ANC } \cdot(\mathrm{ANC} /(\mathrm{ANC}+\mathrm{ANC} 50)) \\
& \cdot(1-\mathrm{B} /(\mathrm{B}+\mathrm{B} 50)) \cdot \mathrm{B} .
\end{aligned}
$$

The term in Eq. (5) describes the bacterial kill by the immune system which is dependent on both the number of neutrophils and the bacterial burden. $\mathrm{B}$ is the bacterial concentration $\left(\mathrm{CFU} \mathrm{m}{ }^{-1}\right)$, Kkill_ANC describes the maximum rate at which neutrophils can kill the pathogens $\left(1.74 \mathrm{~h}^{-1}\right)$, ANC is the absolute neutrophil count (here assumed a constant value of 2500 cells $\mu \mathrm{l}^{-1}$ ), ANC50 is the number of neutrophils required to achieve $50 \%$ of maximum killing by immune cells ( 190.8 cells $\left.\mu \mathrm{l}^{-1}\right)$, and B50 is concentration of bacteria required to saturate the immune cell response by $50 \%\left(430 \times 10^{4} \mathrm{CFU} \mathrm{ml}{ }^{-1}\right)$. Using this model, the bacterial killing by the immune cells will be according to a first order process (according to Kkill_ANC) at low bacterial concentrations. However, when approaching higher bacterial concentrations saturation will occur and the killing will approach a zero order process (constant killing rate). The immune response was assumed to affect all bacteria similarly, regardless of the susceptibility and growth state of the bacteria (see online appendix).

\section{Software}

Perl speaks NONMEM (PsN) was used to facilitate execution, to summarize results and to evaluate the models. A 
more complex model was selected when the difference in the Objective Function Value reported by NONMEM reduced at least 10.83 units $(p<0.001$, for one degree of freedom). To evaluate the typical trends predicted by the model, as well as the model's ability to describe the variability, prediction corrected Visual predictive checks (VPCs) were performed. Piraña, Xpose and R (ggplot) were also used for plotting and analyzing the results from different models and predictions [39-42].

\section{Results}

\section{Development of the WB-PBPK model for ciprofloxacin}

A WB-PBPK model characterizing both the typical trends and variability of the available ciprofloxacin plasma concentration data was successfully developed as illustrated in the VPC (Fig. 2). Inclusion of the clinical Kp values as priors was needed to stabilize the WB-PBPK model and the parameter estimates including clearance and tissue $\mathrm{Kp}$ values were comparable to the literature values (Table 1). The estimate for the $\mathrm{Kp}$ value in muscle deviate the most (except for "Rest") and was estimated to be $60 \%$ higher than the literature prior value. The renal clearance was for a typical patient with a CRCL of $110 \mathrm{ml} \mathrm{min}^{-1}$, estimated to be $49 \%$ of the total clearance, while for a patient with a CRCL of $50 \mathrm{ml} \mathrm{min}^{-1}$ renal clearance was predicted to constitute $13 \%$ of the total clearance. The final model included IIV for clearance and for $\mathrm{Kp}$ values (a common value for all).

\section{Model predictions of unbound concentrations}

Profiles of unbound ciprofloxacin concentration in different tissues were predicted from the developed WB-PBPK model (Fig. 3). Kidney and lung were predicted to have higher exposures as compared to the other organs while muscle, brain and adipose were predicted to have relatively low exposures to ciprofloxacin. Cmax was achieved at the end of the $1 \mathrm{~h}$ constant rate infusion in plasma as well as in all other tissues and organs, depicting the rapid distribution of ciprofloxacin.

\section{Combining the WBPBPK with the PKPD model}

When the predicted PK profile of unbound ciprofloxacin from the WB-PBPK model was driving the PKPD model, the rate and extent of take-over of mutant bacteria in different tissues could be predicted and compared. For kidney, representing a scenario of pyelonephritis, the $400 \mathrm{mg}$ b.i.d. dosing regimen was predicted to be high enough to clear the bacterial population of not only wild type but also mutants of $E$. coli with intermediate level of resistance (i.e. MIC $\left.<10 \mathrm{mg} \mathrm{l}^{-1}\right)$ (Fig. 4a, b). However the highly resistant strains $\left(\mathrm{MIC}>25 \mathrm{mg} \mathrm{l}^{-1}\right.$ ) were not killed off despite the high ciprofloxacin exposures in kidney (Fig. 4c, d).

Similarly, when the bacteria time-course of the $E$. coli strain LM625 (MIC $=0.50 \mathrm{mg}^{-1}$ ) was predicted with the different tissue exposures the bacteria was eliminated from the brain, muscle and skin while re-growth was evident in adipose tissue (Fig. 5).

\section{Addition of neutrophil effect on WBPBPK-PKPD model}

Lastly the effect of neutrophils was added to the model and predictions were made for the wild type and the most resistant $E$. coli strain, LM707 (MIC $\left.=48 \mathrm{mg} \mathrm{l}^{-1}\right)$ in lung and kidney. For the wild-type bacteria, it was predicted that the combination of high exposures of ciprofloxacin and bacterial killing by neutrophils will quickly result in total bacterial eradication. For the resistant strain, the combination was predicted to eliminate the resistant bacteria from kidney in contrast to ciprofloxacin exposure alone (Fig. 6a). In lung, which had the second highest exposure of ciprofloxacin relative to kidney, even adding the effect of immune response was not enough to eliminate the resistant strain and the model predicted re-growth (Fig. 6b).

\section{Discussion}

A WB-PBPK model was successfully developed based on plasma concentration data of ICU patients. The model did not only capture the trends in the observed data but also described the variability in the data as shown in the VPC (Fig. 2). By using clinical Kp values for different tissues as priors a full WB-PBPK model could be developed based on plasma concentration data only. A similar strategy has also been successfully applied for CMS and colistin [43].

This work is the first example of a WB-PBPK model for ciprofloxacin which was implemented using non-linear mixed effects modeling and frequentist priors (\$PRIOR in NONMEM). Using informative priors on clinical Kp values, and information on blood flows and tissue volumes from the literature, the full WB-PBPK model could weigh the individual information from the available plasma concentration data to characterize the $\mathrm{PK}$ in the study population. Using priors is a good way to provide supportive information from earlier studies and to stabilize a WBPBPK model in NONMEM [33].

Estimated $\mathrm{Kp}$ values were in good agreement with the priors (Table 1). There are some notable variations; e.g. 
Fig. 2 Visual predictive check for the WB-PBPK model. Dotted red lines are the median and the 5th and 95th percentiles of the observed plasmaconcentration data. Shaded areas represent the $95 \%$ confidence interval around the median and the 5th and 95th percentiles based on simulations from the model $(n=500)$ (Color figure online)

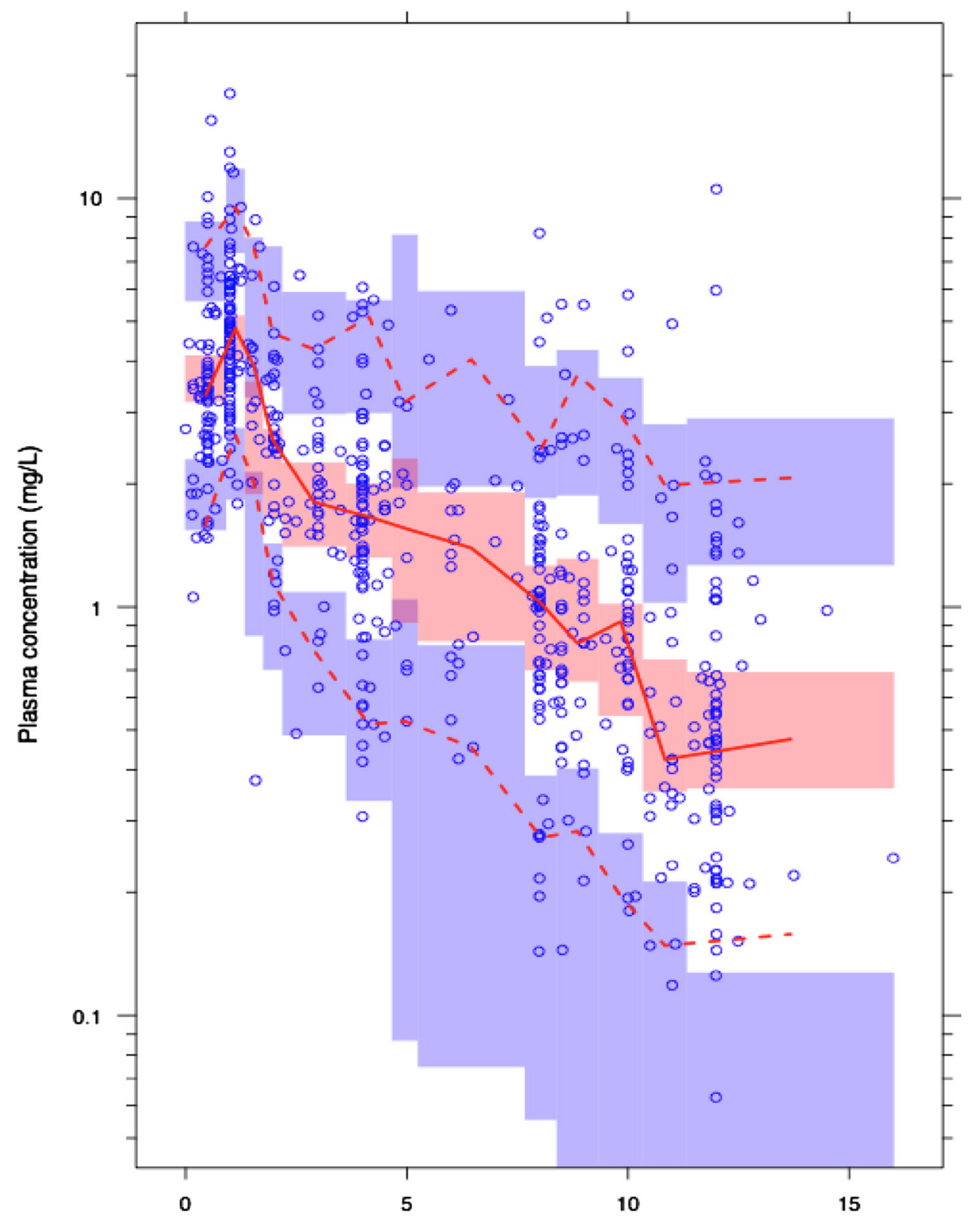

Time after dose (h) muscle, which is a relatively big tissue compartment, had a $60 \%$ higher $\mathrm{Kp}$ estimate as compared to what has been reported in the literature. One reason could be the relatively bigger size of muscle in comparison to other tissues, and a deviation from the prior can thereby have a clear impact on the PK profile. Kp values for relatively small organs e.g. spleen and pancreas were unchanged from the prior, reflecting that either there is not enough information in the data to drive them away from the prior values or estimates and observed values are well in agreement. Also the estimated lung and kidney $\mathrm{Kp}$ values relies primarily on the experimentally determined $\mathrm{Kp}$ values rather than on the plasma concentration data since the deviations from the prior was small, and the uncertainty was the same. Furthermore, there have been some indications of involvement of active efflux transporters, e.g. P-gp and BCRP, on ciprofloxacin distribution in different tissues. There is however limited evidence of any significant impact of these active transporters in vivo for ciprofloxacin [44, 45]. Due to lack of specific data the current model does not account for active processes and perfusion limited drug distribution was assumed in all tissues. Renal clearance was dependent on the kidney function and with decreased CRCL of $50 \mathrm{ml} \mathrm{min}^{-1}$ renal clearance was reduced to $16 \%$ of total clearance. 
Table 1 Prior values applied based on clinical data from the literature and the here estimated parameters of the developed WB-PBPK model

\begin{tabular}{llcc}
\hline & Prior values normal scale (uncertainty) & Model estimate log-scale $( \pm$ SE) & Model estimate normal scale $(\mathrm{RSE})$ \\
\hline $\mathrm{CL}_{\mathrm{NR}}\left(1 \mathrm{~h}^{-1}\right)$ & $7.17(25 \%)$ & $2.60 \pm 0.14$ & 13.5 \\
$\mathrm{f}_{\text {secretion }}$ & $0.57(25 \%)$ & & $0.674(26 \%)$ \\
Kp,lung & $3.3(25 \%)$ & $-0.257 \pm 0.25$ & 3.32 \\
Kp,brain & $0.771(25 \%)$ & $1.30 \pm 0.25$ & 3.67 \\
Kp,heart & $3.67(25 \%)$ & $-0.335 \pm 0.24$ & 0.715 \\
Kp,skin & $0.718(25 \%)$ & $-0.0229 \pm 0.16$ & 0.977 \\
Kp,muscle & $1.6(25 \%)$ & $-0.885 \pm 0.23$ & 0.413 \\
Kp,adipose & $0.449(25 \%)$ & $0.668 \pm 0.25$ & 1.95 \\
Kp,spleen & $1.954(25 \%)$ & $1.21 \pm 0.23$ & 3.35 \\
Kp,GIT & $3.39(25 \%)$ & $1.27 \pm 0.23$ & 3.56 \\
Kp,liver & $3.67(25 \%)$ & $2.09 \pm 0.25$ & 8.09 \\
Kp,kidney & $8.2(25 \%)$ & $1.35 \pm 0.11$ & 3.86 \\
Kp,rest & $2.77(25 \%)$ & & $56(9.3 \%)$ \\
IIV CL $(\mathrm{CV} \%)$ & - & & $55(15 \%)$ \\
IIV Kp (CV \%) & - & & $33(7.1 \%)$ \\
Proportional residual error $(\%)$ & - & & \\
\hline
\end{tabular}

$\mathrm{f}_{\text {secretion }}$ and $\mathrm{CL}_{\mathrm{NR}}$ represent the fraction of ciprofloxacin renal clearance which relies on secretion into renal tubules and non-renal clearance, respectively

Fig. 3 Predicted extracellular tissue concentration-time profiles of unbound ciprofloxacin following a dosing regimen of $400 \mathrm{mg}$ b.i.d
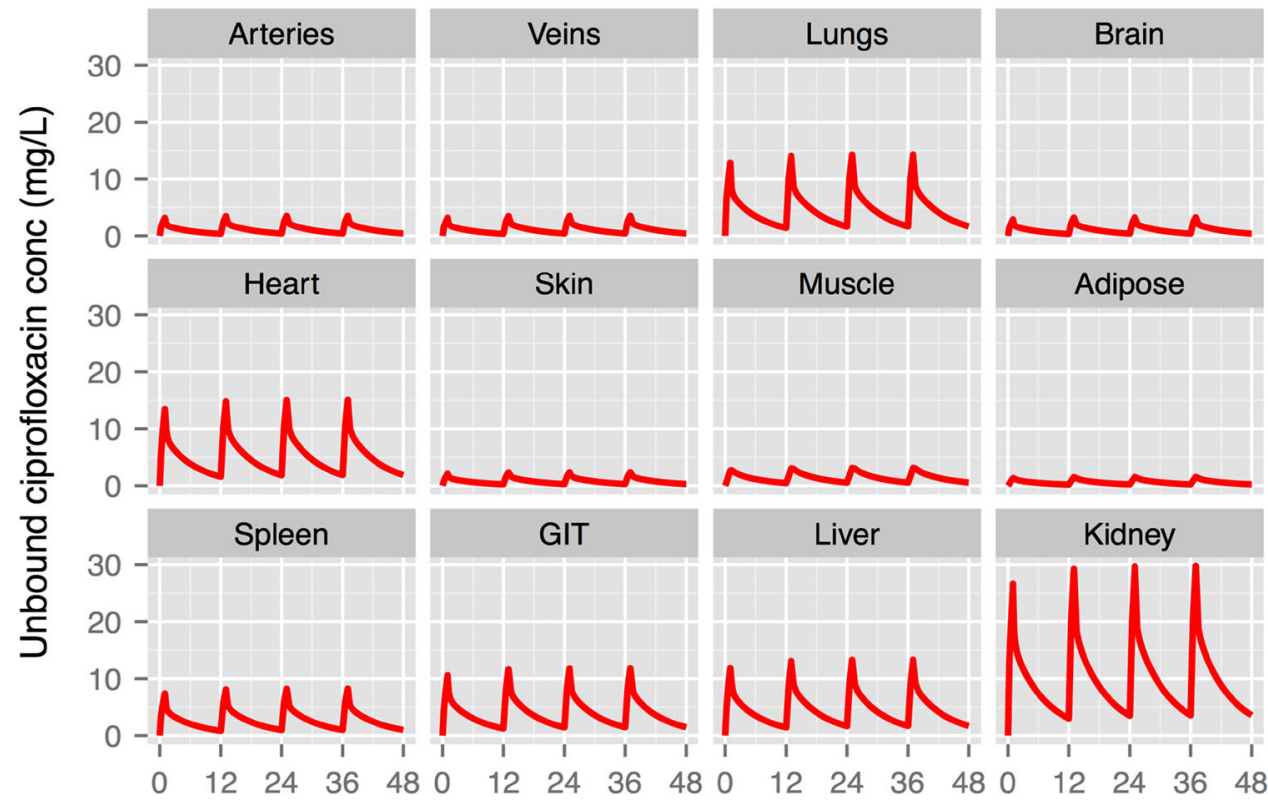

Adipose
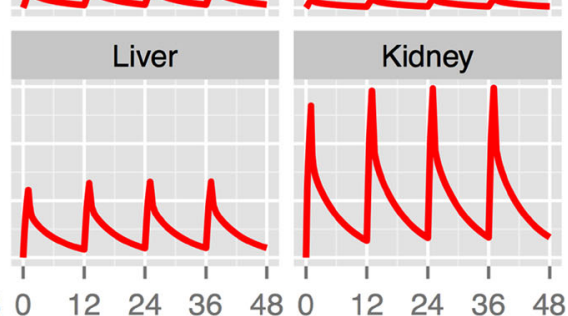

Time (h)

Based on the developed model, the concentration time profile of ciprofloxacin was predicted in different tissues. The highest unbound concentrations were observed in kidney and lung, which is in line with the ciprofloxacin treatment indications of pneumonia and urinary tract infections. The NONMEM implementation allowed for connecting the WB-PBPK model to an in vitro based PKPD model, where the predicted unbound concentration in extracellular fluid was driving the bacterial killing. It was also illustrated how a function for the immune response could be added to investigate the impact on the rate and extent of bacterial killing.

During recent years, the use of PBPK models has become an integral tool in drug development. Here we suggest a framework in which a WB-PBPK model is combined with a PKPD model that can describe the time- 
Fig. 4 Predictions of the time course of bacterial killing of different $E$. coli strains in the extracellular compartment of kidney following a ciprofloxacin dose of $400 \mathrm{mg}$ b.i.d. Grey lines represents the resistant bacterial strain while black lines represent wild type bacterial strain (LM347, MIC $=0.023 \mathrm{mg}^{-1}$ ). Please note that in $\mathrm{A}$ the two strains are overlapping
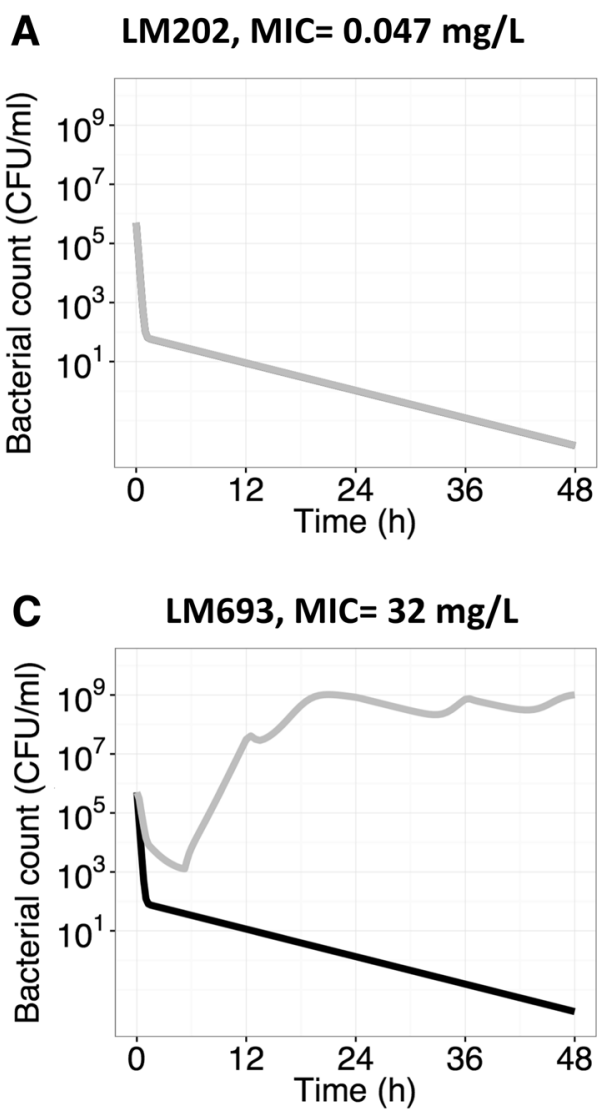

Fig. 5 Predictions of the time course of bacterial killing of E. coli strains LM347 (black) and LM625 (grey) in the extracellular compartment of different tissues (brain, muscle, adipose and skin) following a ciprofloxacin dose of $400 \mathrm{mg}$ b.i.d
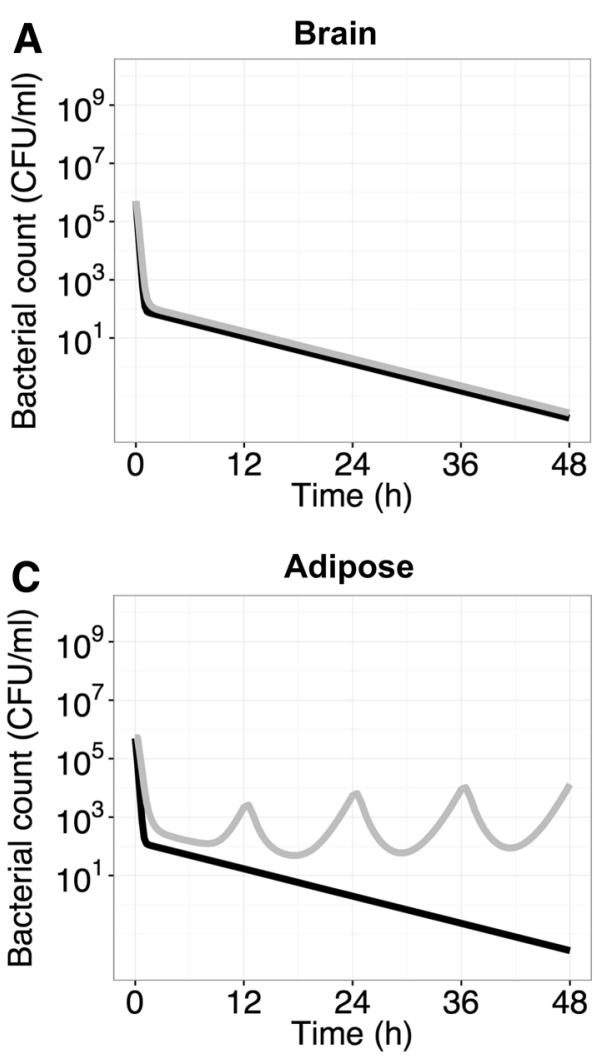

\section{B $\quad L M \quad 625, M I C=0.5 \mathrm{mg} / \mathrm{L}$}
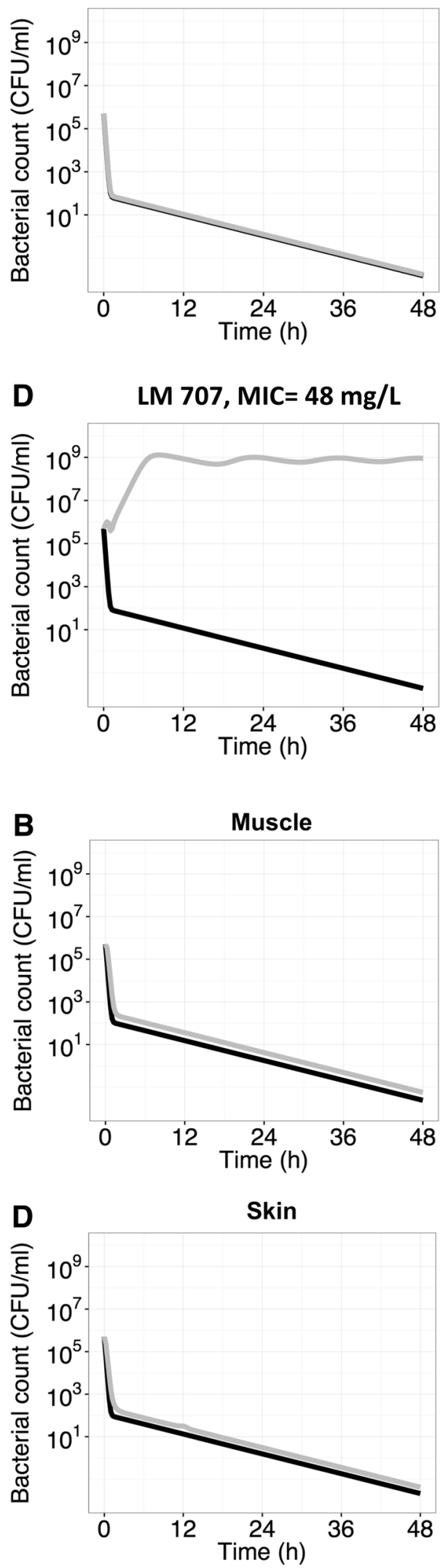
Fig. 6 Predictions of the time course of bacterial killing of E. coli strains LM347 (black) and LM707 (grey) in lung and kidney following administration of ciprofloxacin $400 \mathrm{mg}$ b.i.d. with (dashed lines) and without (solid lines) addition of function for immune response
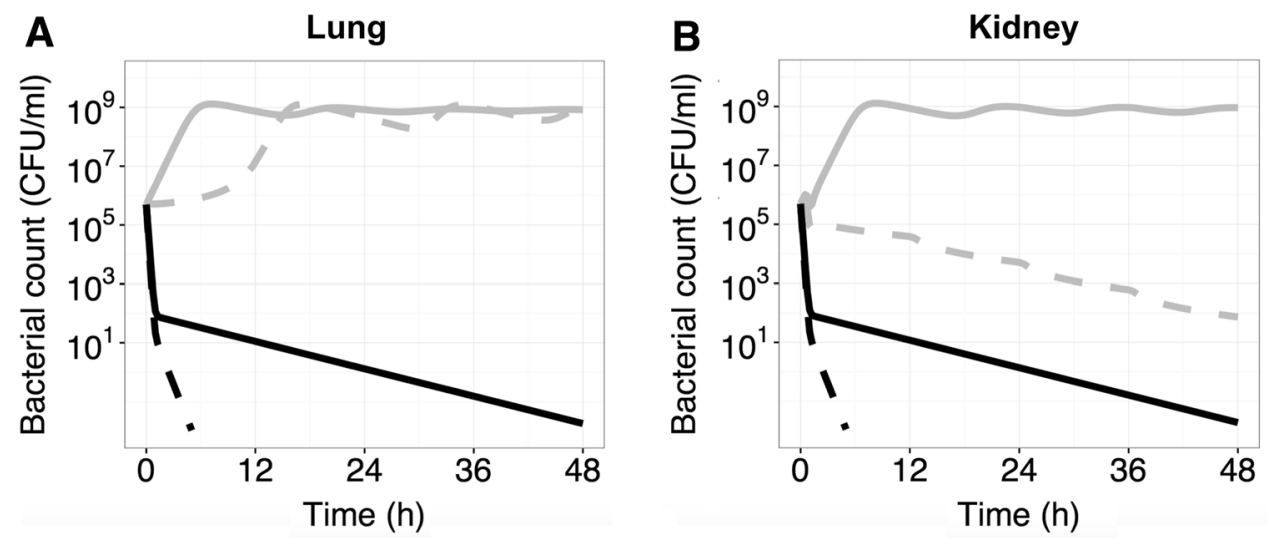

course of bacterial growth and kill. Thereby the relative kill of different dosing regimens and bacterial strains can be compared for different tissues. In the in vitro PKPD model drug concentration in the test tube was used to drive the bacterial killing while here the predicted unbound drug concentration-time profile in different tissues was replacing the static test tube concentration in the $\mathrm{E}_{\max }-$ model. Similar predictions based on unbound plasma concentrations in plasma have earlier been used to support treatment regimens of e.g. gentamicin, colistin and meropenem [46].

It should be noted that there are several assumptions in the predictions illustrated. For example, the predictions assume that in vivo bacterial growth and killing with the antibacterial will be similar to the in vitro test tube situation. This work is however one step closer to the in vivo situation compared to the test tube situation as we use the predicted time course of unbound ciprofloxacin at potential infection sites based on clinical data. The predicted concentration-time profiles are most likely a better representation of the concentration in the extracellular tissue fluids than the plasma concentration time-profiles that are typically used to illustrate bacterial killing in in vitro kinetic systems. This modeling framework illustrates a way of combining PK and PKPD information from in vitro, in vivo and clinical data to make a best guess. Once the separate model components have been developed and connected, different scenarios can be simulated and different hypothesis be tested. For example, the framework could be applied for comparing dosing regimens between different study populations with different PK, and different types of bacteria and/or infection sites. The model could also be used to explore the potential for new indications, i.e. different infection sites where the drug concentrations are predicted to be sufficiently high for a good bactericidal effect. Here we explored the bacterial killing using different bacterial strains with different degree of drug susceptibility, but also the impact of e.g. a lower fitness or growth rate in the tissues could be tested.
The immune response is an important factor for the outcome of an antibacterial therapy. An extreme example of the complications with immunodeficiency is HIV patients who require higher doses and combination antibiotic treatment as compared to an immunocompetent patients [47, 48]. Most often the efficacy of antibacterials is estimated based on the data from in vitro time kill curve experiments and/or in vivo studies using neutropenic mice, thus ignoring the role of neutrophils and other immune cells. Recently, Lyons et al. described a physiologically based PKPD model of rifampin therapy in a mouse tuberculosis infection model also accounting for dynamics for host immune response to Mycobacterium tuberculosis infection [49]. In this work we wanted to also illustrate how this type of framework can be used to explore the relative response of the immune system and drug treatment. With the addition of immune response, the highly resistant strain, LM707, was predicted to be eliminated from kidney while in lung, which had slightly lower levels of ciprofloxacin, addition of an immune response was not enough to eliminate the bacteria (Fig. 6b). The equation applied for the immune response was based on two in vivo studies quantifying the effect of neutrophils and bacterial burden (Eq. 5). It should be noted that these predictions are based on the effect as quantified in mice experiments and that further studies to elucidate the quantitative impact of the immune cells are warranted.

In this project we suggest a framework where data from different sources and different models are integrated to simulate the time course of unbound concentration and bacterial killing at the site of drug action. This approach represents a possibility to predict the therapy outcome in different patient populations by combining the information from different sources. In its present state this framework is based on strong assumptions as, for example, there is limited information available on possible differences in bacterial growth rates and inoculum sizes at different infection sites, and data on the impact of the immune system is sparse. Despite its limitations it represents a show 
case and a step in the direction of model-informed drug discovery and development (MID3) [11] by incorporating information from several sources and the application of translational approaches. This type of WB-PBPKPD modeling framework has thereby the potential to help guiding drug development of antimicrobials. The approach can especially be valuable given that we are rapidly facing an era of increasing resistance, where there is an urgent need for new antimicrobial agents and effective drug combinations. Given the parameterization of the WBPBPK model into volumes and flows, the framework can also be extended and adapted to other patient populations where differences in drug disposition is expected, such as for pediatric and septic shock patients.

\section{Conclusion}

The developed WB-PBPK model successfully described the plasma concentration profile data from patients by characterizing both typical trends and variability where parameter estimation was based on plasma concentrations combined with prior information on ciprofloxacin $\mathrm{Kp}$ values and clearance. Linking the predicted unbound extracellular concentration-time profiles from the WB-PBPK model with a PKPD model developed based on in vitro data is a promising approach for predicting infection site specific time courses of bacterial killing. This type of framework can be applied to support treatment strategies for selection of antibiotics for different indications and investigate the impact of different levels of drug susceptibility for the bacteria.

Acknowledgments This work was supported by the Swedish Foundation for Strategic Research and DDMoRe which is an Innovative Medicines Initiative Joint Undertaking under Grant Agreement No. 115156 , resources of which are composed of financial contributions from the European Union's Seventh Framework Programme (FP7/ 2007-2013) and EFPIA companies' in kind contribution. The DDMoRe project is also supported by financial contribution from Academic and SME partners. This work does not necessarily represent the view of all DDMoRe partners. Drs. David Khan and Salim Bouchene are acknowledged for their support in implementation of the WB-PBPK-PD model.

Open Access This article is distributed under the terms of the Creative Commons Attribution 4.0 International License (http://creative commons.org/licenses/by/4.0/), which permits unrestricted use, distribution, and reproduction in any medium, provided you give appropriate credit to the original author(s) and the source, provide a link to the Creative Commons license, and indicate if changes were made.

\section{References}

1. Jones HM et al (2015) Physiologically based pharmacokinetic modeling in drug discovery and development: a pharmaceutical industry perspective. Clin Pharmacol Ther 97(3):247-262
2. Tulkens PM (1991) Intracellular distribution and activity of antibiotics. Eur J Clin Microbiol Infect Dis 10(2):100-106

3. Nielsen EI, Friberg LE (2013) Pharmacokinetic-pharmacodynamic modeling of antibacterial drugs. Pharmacol Rev 65(3): 1053-1090

4. Gerlowski LE, Jain RK (1983) Physiologically based pharmacokinetic modeling: principles and applications. J Pharm Sci 72(10):1103-1127

5. Theil FP et al (2003) Utility of physiologically based pharmacokinetic models to drug development and rational drug discovery candidate selection. Toxicol Lett 138(1-2):29-49

6. Rowland M (2013) Physiologically-based pharmacokinetic (PBPK) modeling and simulations principles, methods, and applications in the pharmaceutical industry. CPT Pharmacomet Syst Pharmacol 2:e55

7. Nestorov I (2007) Whole-body physiologically based pharmacokinetic models. Expert Opin Drug Metab Toxicol 3(2):235-249

8. Rowland M, Lesko LJ, Rostami-Hodjegan A (2015) Physiologically based pharmacokinetics is impacting drug development and regulatory decision making. CPT 4(6):313-315

9. Parrott $\mathrm{N}$ et al (2005) An evaluation of the utility of physiologically based models of pharmacokinetics in early drug discovery. J Pharm Sci 94(10):2327-2343

10. Rodgers T, Leahy D, Rowland M (2005) Physiologically based pharmacokinetic modeling 1: predicting the tissue distribution of moderate-to-strong bases. J Pharm Sci 94(6):1259-1276

11. Workgroup EM et al (2016) Good Practices in Model-Informed Drug Discovery and Development: practice, Application, and Documentation. CPT 5(3):93-122

12. Zhao P et al (2011) Applications of physiologically based pharmacokinetic (PBPK) modeling and simulation during regulatory review. Clin Pharmacol Ther 89(2):259-267

13. Rodgers T, Rowland M (2006) Physiologically based pharmacokinetic modelling 2: predicting the tissue distribution of acids, very weak bases, neutrals and zwitterions. J Pharm Sci 95(6): 1238-1257

14. Bjorkman S et al (2001) Prediction of the disposition of midazolam in surgical patients by a physiologically based pharmacokinetic model. J Pharm Sci 90(9):1226-1241

15. Poulin P, Theil FP (2000) A priori prediction of tissue:plasma partition coefficients of drugs to facilitate the use of physiologically-based pharmacokinetic models in drug discovery. J Pharm Sci 89(1):16-35

16. Nielsen EI, Cars O, Friberg LE (2011) Predicting in vitro antibacterial efficacy across experimental designs with a semimechanistic pharmacokinetic-pharmacodynamic model. Antimicrob Agents Chemother 55(4):1571-1579

17. Mohamed AF, Cars O, Friberg LE (2014) A pharmacokinetic/ pharmacodynamic model developed for the effect of colistin on Pseudomonas aeruginosa in vitro with evaluation of population pharmacokinetic variability on simulated bacterial killing. J Antimicrob Chemother 69(5):1350-1361

18. Mohamed AF et al (2011) Pharmacokinetic-pharmacodynamic model for gentamicin and its adaptive resistance with predictions of dosing schedules in newborn infants. Antimicrob Agents Chemother 56(1):179-188

19. Guo B et al (2011) Quantitative impact of neutrophils on bacterial clearance in a murine pneumonia model. Antimicrob Agents Chemother 55(10):4601-4605

20. Drusano GL et al (2010) Impact of burden on granulocyte clearance of bacteria in a mouse thigh infection model. Antimicrob Agents Chemother 54(10):4368-4372

21. Dalhoff A (2012) Global fluoroquinolone resistance epidemiology and implictions for clinical use. Interdiscip Perspect Infect Dis 2012:976273 
22. Khan DD et al (2015) A mechanism-based pharmacokinetic/ pharmacodynamic model allows prediction of antibiotic killing from MIC values for WT and mutants. J Antimicrob Chemother 70(11):3051-3060

23. Khachman D et al (2011) Optimizing ciprofloxacin dosing in intensive care unit patients through the use of population pharmacokinetic-pharmacodynamic analysis and Monte Carlo simulations. J Antimicrob Chemother 66(8):1798-1809

24. Beal S, Sheiner LB, Boeckmann A, Bauer RJ (2009) NONMEM User's Guides (1989-2009). Icon Development Solutions, Ellicott City

25. Davey PG et al (1994) Ciprofloxacin and sparfloxacin penetration into human brain tissue and their activity as antagonists of GABAA receptor of rat vagus nerve. Antimicrob Agents Chemother 38(6): 1356-1362

26. Mertes PM et al (1990) Penetration of ciprofloxacin into heart valves, myocardium, mediastinal fat, and sternal bone marrow in humans. Antimicrob Agents Chemother 34(3):398-401

27. Daschner FD, Westenfelder M, Dalhoff A (1986) Penetration of ciprofloxacin into kidney, fat, muscle and skin tissue. Eur J Clin Microbiol 5(2):212-213

28. Brunner $\mathrm{M}$ et al (2004) [18F] Ciprofloxacin, a new positron emission tomography tracer for noninvasive assessment of the tissue distribution and pharmacokinetics of ciprofloxacin in humans. Antimicrob Agents Chemother 48(10):3850-3857

29. Birmingham MC et al (1998) Ciprofloxacin (CIPRO) concentrations in lung tissue following a single $400 \mathrm{mg}$ intravenous dose. Crit Care Med 26(1):100A

30. Brismar B et al (1990) Ciprofloxacin concentrations and impact of the colon microflora in patients undergoing colorectal surgery. Antimicrob Agents Chemother 34(3):481-483

31. Dan M et al (1987) Concentrations of ciprofloxacin in human liver, gallbladder, and bile after oral administration. Eur J Clin Pharmacol 32(2):217-218

32. Brunner M et al (1999) Distribution and antimicrobial activity of ciprofloxacin in human soft tissues. Antimicrob Agents Chemother 43(5):1307-1309

33. Langdon $\mathrm{G}$ et al (2007) Linking preclinical and clinical wholebody physiologically based pharmacokinetic models with prior distributions in NONMEM. Eur J Clin Pharmacol 63(5):485-498

34. Poulin P, Theil FP (2002) Prediction of pharmacokinetics prior to in vivo studies. II. Generic physiologically based pharmacokinetic models of drug disposition. J Pharm Sci 91(5):1358-1370

35. Basic anatomical and physiological data for use in radiological protection: reference values. A report of age- and gender-related differences in the anatomical and physiological characteristics of reference individuals. ICRP Publication 89. Ann ICRP, 2002. 32(3-4): p. 5-265

36. Davies B, Morris T (1993) Physiological parameters in laboratory animals and humans. Pharm Res 10(7):1093-1095

37. Vance-Bryan K, Guay DR, Rotschafer JC (1990) Clinical pharmacokinetics of ciprofloxacin. Clin Pharmacokinet 19(6):434461

38. McNamara PJ, Gibaldi M, Stoeckel K (1983) Fraction unbound in interstitial fluid. J Pharm Sci 72(7):834-836

39. Ito K, Murphy D (2013) Application of ggplot2 to pharmacometric graphics. CPT Pharmacomet Syst Pharmacol 2:e79

40. Jonsson EN, Karlsson MO (1999) Xpose-an S-PLUS based population pharmacokinetic/pharmacodynamic model building aid for NONMEM. Comput Methods Progr Biomed 58(1):51-64

41. Bergstrand $M$ et al (2011) Prediction-corrected visual predictive checks for diagnosing nonlinear mixed-effects models. AAPS J 13(2):143-151

42. Keizer RJ, Karlsson MO, Hooker A (2013) Modeling and Simulation workbench for NONMEM: tutorial on Pirana, PsN, and Xpose. CPT Pharmacomet Syst Pharmacol 2:e50

43. Bouchene S (2016) Physiologically based pharmacometric models for colistin and the immune response to bacterial infection, in pharmaceutical biosciences. Uppsala University, Uppsala

44. Ong HX et al (2013) Ciprofloxacin is actively transported across bronchial lung epithelial cells using a Calu-3 air interface cell model. Antimicrobial Agents Chemother 57(6):2535-2540

45. Park MS, Okochi H, Benet LZ (2011) Is ciprofloxacin a substrate of P-glycoprotein? Arch Drug Inf 4(1):1-9

46. Mohamed AF et al (2016) Dynamic interaction of colistin and meropenem on a WT and a resistant strain of Pseudomonas aeruginosa as quantified in a PK/PD model. J Antimicrob Chemother 71(5):1279-1290

47. Laxminarayan R et al (2006) Drug Resistance. In: Jamison DT et al (eds) Disease control priorities in developing countries, chap. 55, 2nd edn. The International Bank for Reconstruction and Development/The World Bank, Washington (DC); Oxford University Press, New York

48. Shlaes DM, Binczewski B, Rice LB (1993) Emerging antimicrobial resistance and the immunocompromised host. Clin Infect Dis 17(Suppl 2):S527-S536

49. Lyons MA, Lenaerts AJ (2015) Computational pharmacokinetics/ pharmacodynamics of rifampin in a mouse tuberculosis infection model. J Pharmacokinet Pharmacodyn 42(4):375-389 